\section{Zum Blasenkrebs gesellt sich nicht selten Lungenkrebs}

\author{
Bei Patienten mit einem Harnblasenkarzinom könnte das Screenen auf \\ Lungenkrebs mithilfe einer Niedrigdosis-Computertomografie sinnvoll sein. \\ Das gemeinsame Auftreten beider Krebsarten scheint gar nicht so selten.
}

$\mathrm{R}_{\mathrm{h}}^{\mathrm{a}}$ uchen steht in engem Zusammenhang mit der Entwicklung von Lungen- wie auch Harnblasenkarzinomen. Wie häufig beide Tumorarten isoliert oder gemeinsam auftreten, zeigen zwei aktuelle große US-amerikanische Studien: die PLCO(Prostate, Lung, Colorectal and Ovarian Cancer Screening Trial) -Studie und die NLST(National Lung Cancer Screening Trial)-Studie. Die Urologen Dr. Laura-Maria Krabbe, Münster, und Prof. Yair Lotan, Dallas, USA, haben die Daten genauer untersucht.

An der PLCO-Studie haben fast 155.000 und an der NLST-Studie knapp 53.500 Patienten teilgenommen. Beides sind prospektive Längsschnittstudien.
Insgesamt 1.430 bzw. 3.519 PLCO-Patienten sowie 439 bzw. 2.058 NLST-Patienten sind im jeweiligen Studienzeitraum an Blasen- bzw. Lungenkrebs erkrankt.

Insgesamt 58 Patienten $(0,03 \%)$ der PLCO-Studie und 22 Patienten (0,04\%) der NLST-Studie waren innerhalb des Beobachtungszeitraumes an beiden Tumorarten erkrankt. Das bedeutet, dass in der PLCO- und in der NLST-Studie immerhin 4,1 \% (58/1430) bzw. 5,0\% (22/439) der Blasenkrebspatienten auch Lungenkrebs und 1,7 \% (58/3519) bzw. $1,1 \%(22 / 2058)$ der Lungenkrebspatienten auch Blasenkrebs hatten. Die meisten der Doppelerkrankten waren weiße Männer und/oder Raucher mit mindes- tens 30 Packungsjahren (ein Packungsjahr entspricht bspw. 20 Zigaretten pro Tag ein Jahr lang oder zehn pro Tag über zwei Jahre). Sie waren zum Zeitpunkt der Studien aktive Raucher oder hatten frühestens 15 Jahre vor Eintritt in die jeweilige Studie aufgehört.

Fazit: Da das Gesamtrisiko, an Lungenkrebs zu erkranken, bei den Teilnehmern der beiden Studien mit Blasenkrebs zwischen $4 \%$ und $5 \%$ liegt und damit deutlich höher als in der Allgemeinbevölkerung - in diesem Jahr werden in Deutschland voraussichtlich etwa 55.000 Menschen ein Bronchialkarzinom entwickeln -, halten die Urologen es für sinnvoll, ihre Kollegen zu ermuntern, Patienten mit Blasenkrebs mithilfe einer Niedrigdosis-CT-Aufnahme des Thorax auch auf Lungenkrebs zu untersuchen. Peter Leiner

Krabbe LM et al. Should patients newly diagnosed with bladder cancer be screened for lung cancer? Int J Urol. 2016;doi: 10.1111/iju.13052.

\section{Gute Prognose bei inzidentellem Prostatakarzinom}

\section{Karzinome, die zufällig bei einer transurethralen Resektion der Prostata zutage gefördert werden, führen seltener als andere lokal begrenzte Prostatakarzinome in den ersten zehn Jahren zum Tod.}

W ird ein Prostatakarzinom nur infolge einer transurethralen Resektion der Prostata (TURP) diagnostiziert, besteht die Gefahr, dass der Tumor nicht vollständig entfernt wurde. Eine radikale Prostatektomie ist daher zumindest in den europäischen Leitlinien optional vorgesehen, sofern die Patienten noch eine Lebenserwartung von mehr als zehn Jahren haben.

Die Angst, inzidentelle Prostatakarzinome (IPC) könnten besonders rasch progredient sein, scheint allerdings unbegründet. Nach Daten aus dem nationalen Krebsregister Englands liegt die krebsspezifische 10-Jahres-Mortalität von lokal begrenzten IPC um $30 \%$ niedriger als bei anderen lokalisierten Prostatakarzinomen.
Die Daten stammen von 192.960 Männern, die zwischen 2000 und 2008 wegen einer Prostatakrebsdiagnose registriert und im Median 4,7 Jahre nachbeobachtet worden waren. Ausgewählt wurden nur Patienten mit T1- und T2-Tumoren, die keine Metastasen hatten und nicht in den ersten sechs Monate gestorben waren. 6.666 Männer (3,5\%) litten unter einm IPC. Sie hatten tendenziell ein höheres Alter, weniger Begleiterkrankungen und wurden seltener einer radikalen Operation unterzogen als die übrigen $\mathrm{Pa}$ tienten. Trotzdem war ihre krebsspezifische Zehn-Jahres-Mortalität mit 17,1 \% signifikant geringer als die von NichtIPC-Patienten mit 19,0\%. Wurden die Unterschiede in Alter, Begleiterkrankungen und Behandlung berücksichtigt, trat der Vorteil noch deutlicher zutage. IPCPatienten hatten dann ein um $30 \%$ niedrigeres relatives Risiko, innerhalb von zehn Jahren an den Folgen des Prostatakrebses zu sterben.

Die 10-Jahres-Gesamtmortalität war unbereinigt in der IPC-Gruppe höher (49,2 \% vs. 40,1 \%). Wenn Patientencharakteristika und Behandlungsstrategien abgeglichen wurden, war jedoch kein Unterschied mehr zu erkennen.

Fazit: Die Studienautoren um Eleni Anastasiadis, Royal College of Surgeons of England, raten trotz der relativ günstigen Prognose von IPC zu weiteren Untersuchungen. Beim IPC handele es sich um eine „variable Erkrankung“. Um eine Erkrankung in der Restprostata auszuschließen, könnten zusätzliche Biopsien oder der Einsatz der Multiparameter-MRT von Nutzen sein.

\section{Dr. Beate Schumacher}

Anastasiadis E et al. Incidental prostate cancer diagnosed following a transurethral resection of the prostate: A national database analysis in England. J Clin Urol 2015;doi: $10.1177 / 2051415815603275$ 